\title{
Tumor necrosis factor antagonists in the treatment of multicentric reticulohistiocytosis: Current clinical evidence
}

\author{
HONGJUN ZHAO ${ }^{1}$, CHUNMEI WU $^{1}$, MENGYUN WU ${ }^{1}$, YAOU ZHOU ${ }^{1}$, HONGLIN ZHU $^{1}$, \\ YISHA LI ${ }^{1}$, YUNHUI YOU ${ }^{1}$, HUI LUO $^{1}$, LIJING WANG ${ }^{2}$ and XIAOXIA ZUO ${ }^{1}$ \\ Departments of ${ }^{1}$ Rheumatology and Immunology, and ${ }^{2}$ Gerontology, Xiangya Hospital, \\ Central South University, Changsha, Hunan 410008, P.R. China
}

Received May 7, 2015; Accepted April 4, 2016

DOI: $10.3892 / \mathrm{mmr} .2016 .5253$

\begin{abstract}
Multicentric reticulohistiocytosis (MRH) is a rare and debilitating systemic disorder characterized by cutaneous nodules and destructive polyarthritis. Due to its unknown etiology, the treatment of MRH varies with different rates of success, which causes treatment options to be rather independent and empirical. In the present study, a case of a 48-year-old woman with a 12-month history of polyarthralgia and skin nodules was reported. Biopsy samples, which were obtained from her skin eruption exhibited dermal infiltration with histiocytes and multinucleated giant cells. Immunohistochemical staining indicated positivity for CD68. The patient was diagnosed with MRH and treated with a combination therapy of infliximab, prednisolone and methotrexate. Her symptoms improved markedly within 2 weeks. Following the results of this case study, a systematic review of 17 cases of MRH treated with tumor necrosis factor (TNF) antagonists was performed, and the efficacy of anti-TNF treatment in MRH was analyzed.
\end{abstract}

\section{Introduction}

Multicentric reticulohistiocytosis (MRH) is a rare, multisystem inflammatory disease, which is characterized by cutaneous nodules and destructive polyarthritis. It can affect any organs or tissues, however, the most common clinical manifestations are papulonodular eruptions and symmetric inflammatory polyarthritis. It is possible to observe constitutional symptoms, including fever, weight loss and malaise,

Correspondence to: Dr Hui Luo, Department of Rheumatology and Immunology, Xiangya Hospital, Central South University, 87 Xiangya Road, Changsha, Hunan 410008, P.R. China

E-mail: huiluo2015@sina.com

Dr Lijing Wang, Department of Gerontology, Xiangya Hospital, Central South University, 87 Xiangya Road, Changsha, Hunan 410008, P.R. China

E-mail: lijingwang2015@sina.com

Key words: muticentric reticulohistiocytosis, tumor necrosis factor- $\alpha$, anti-tumor necrosis factor- $\alpha$ agents, infliximab which may be associated with joint and skin symptoms. There are no specific laboratory tests for the diagnosis of $\mathrm{MRH}$, and its current diagnosis is predominantly dependent on histopathological evaluation $(1,2)$. According to tissue biopsies of the affected areas, ground-glass opacity with increased quantities of periodic acid Schiff-positive materials can be observed, which indicates the infiltration of typical mononuclear histiocytes and multinucleated giant cells (1). In the case of MRH, immunohistochemical analyses are usually positive for CD45, CD68 and HLA-DR, but are negative for S-100, a Langerhans dendritic cell marker, and HHF-35 actin, a fibroblast marker $(1,3)$. In addition, it has been found that serum levels of cytokines, including tumor necrosis factor (TNF)- $\alpha$ and interleukins (ILs), including IL-1 $\beta$, IL-6 and IL-8, are increased in MRH and decreased upon successful

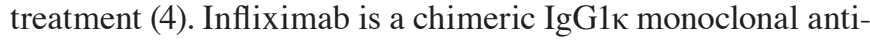
body, which is specific for human TNF- $\alpha$. It is widely used for the treatment of rheumatoid arthritis, ankylosing spondylitis, psoriasis, psoriatic arthritis, Crohn's disease and ulcerative colitis (5). Previously, following the demonstration of increased levels of TNF- $\alpha$ in patients with MRH, anti-TNF- $\alpha$ treatment has been adopted with promising results $(4,6)$.

The present study reported on a case of a patient with MRH, whose arthralgia and skin eruptions significantly regressed following a treatment regimen combining infliximab, prednisolone and methotrexate (MTX). This outcome demonstrated the effectiveness of anti-TNF- $\alpha$ therapy for MRH. A systematic review of available literature was also performed to evaluate the efficacy of anti-TNF- $\alpha$ agents in the treatment of MRH.

\section{Materials and methods}

The present study was approved by the ethics committee of Xiangya Hospital, Central South University (Changsha, China). In 2013, a 48-year-old female diagnosed with MRH, who had a 12-month history of weakness, polyarthralgia, morning stiffness and papulonodular skin eruption was recruited. Her past medical history was unremarkable. According to the results of a biopsy, her skin eruption exhibited dermal infiltration with histiocytes and multinucleated giant cells.

Immunohistochemical staining was performed on samples from the left face cutaneous nodules. Samples were 
fixed using $10 \%$ formalin, embedded in paraffin and cut into $0.25-0.30 \mathrm{~mm}$ sections. Immunohistochemical staining was conducted at room temperature on a shaker. To enhance tissue penetration by antibodies, sections were incubated with ethanol for $30 \mathrm{~min}$ and rinsed with phosphate-buffered saline (PBS) 3 times for $5 \mathrm{~min}$ then blocked to prevent nonspecific primary antibody reactions with $10 \%$ normal donkey serum (NDS; OriGene Technologies, Inc., Beijing, China). Tissue sections were incubated overnight in anti-S-100 (cat. no. MAB-0697) and CD68 (cat. no. MAB-0041) primary antibodies (OriGene Technologies, Inc.). After reaction completion, tissues were rinsed with PBS (3 times for $5 \mathrm{~min}$ ), treated with NDS for $15 \mathrm{~min}$, and incubated with goat anti-mouse fluorescein isothiocyanate-conjugated secondary antibody (cat. no. PV-6000; OriGene Technologies, Inc.) for $3 \mathrm{~h}$, rinsed with PBS, and mounted with Vectashield. The dilutions used were optimal, according to the manufacturer's recommendations. Images were acquired using a cooled CCD camera attached to a light microscope The results of immunnohistochemical staining indicated positivity for CD68. The patient was treated with combination therapy of infliximab (intravenous infusion of $200 \mathrm{mg}$ and subsequent infusion at weeks 2 and 6 , followed by an infusion once every 8 weeks; Cilag AG, Schaffhausen, Switzerland), prednisolone (oral administration; 30 mg/day; Zhejiang Xianju Pharmaceutical Co., Ltd., Zhejiang, China) and MTX (15 mg/week; Shanghai Sine Pharmaceutical Laboratories Co.,Ltd., Shanghai, China).

In addition to the above-mentioned patient, a systematic review was performed on the therapeutic application of anti-TNF- $\alpha$ agents in MRH. This involved the analysis of articles published in the PubMed database (www.ncbi.nlm.nih.gov/pubmed) between January 2003 and April 2014, and additional references cited in these articles were cross-checked. The search strategy involved the use of a combination of key words, including 'Multicentric reticulohistiocytosis', 'Infliximab', 'Etanercept' and 'Adalimumab'.

\section{Results}

Case study. In the case of the female patient recruited in the present study, physical examination revealed an erythematous papulonodular rash, which had developed across her face, anterior chest, back neck, forearms and the dorsum of her fingers, with sizes ranging between 3 and $8 \mathrm{~mm}$ in diameter (Fig. 1). Musculoskeletal examination revealed swelling and tenderness at the joints of the patient's hands, elbows and knees. Initial investigations revealed normal full blood counts, blood lipids, C-reactive protein and erythrocyte sedimentation rate. Anti-nuclear antibodies, rheumatoid factor, anti-neutrophil cytoplasmic antibodies, tumor markers [cancer antigen (CA)199, CA125, CA242, CA153, carcinoembryonic antigen, neuron-specific enolase and $\alpha$-fetoprotein] and tuberculosis antibodies were all negative. In addition, gynecological examination and breast ultrasound were performed to exclude the possibility of gynecological malignancy. Bone marrow aspiration was also performed to rule out the possibility of hematologic neoplasms. Hand X-ray revealed marginal erosions in certain areas of the proximal interphalangeal joints, accompanied with mild osteoporosis and knee

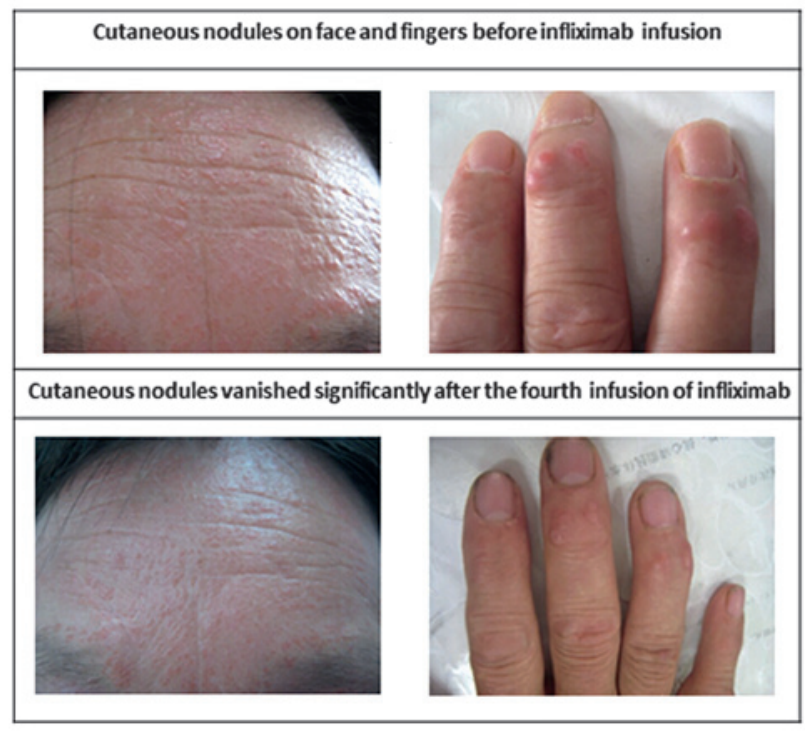

Figure 1. Nodule improvement. Images prior to and following infusion.

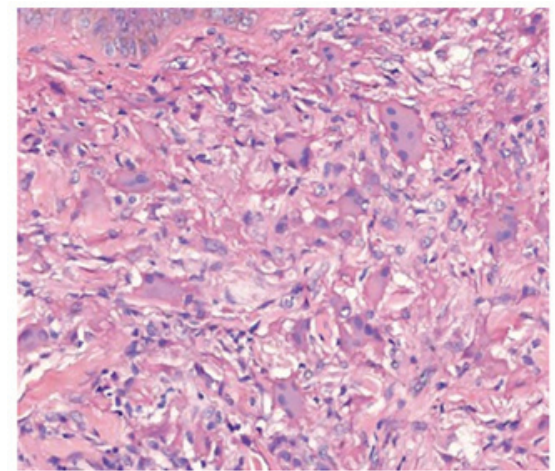

Figure 2. Hematoxylin and eosin staining of left face cutaneous nodules biopsy exhibiting dermal infiltration with histiocytes and multinucleated giant cells (magnification, $\mathrm{x} 400$ ).

osteoarthritis. The chest X-ray findings suggested the possibility of tuberculosis, which was excluded by high-resolution computed tomography scan. Biopsy samples from two of the skin nodules exhibited dermal infiltration with histiocytes and multinucleated giant cells (Fig. 2). Immunohistochemical staining showed suspected positivity for CD68, but negativity for S-100 (Fig. 3). These findings were particularly indicative of MRH.

The patient was initially treated with an infliximab infusion of $200 \mathrm{mg}$, and subsequent infusions were administered at weeks 2 and 6 , followed by an infusion once every 8 weeks. The infliximab infusion was combined with oral prednisolone (30 mg/day), leflunomide (20 mg/day), hydroxychloroquine (HCQ; $200 \mathrm{mg} /$ day), and intravenous MTX (15 mg/week). Diacerein $(50 \mathrm{mg} /$ day) was added to the regimen due to osteoarthritis. The patients symptoms improved following treatment for 3 days. Gradual remission of the erythematous papules and nodules were noted prior to the second infusion of infliximab, and polyarthralgia and stiffness were markedly reduced. Following the fifth infusion, the skin signs had regressed markedly (Fig. 1), and symptoms of arthralgia were no longer present. 


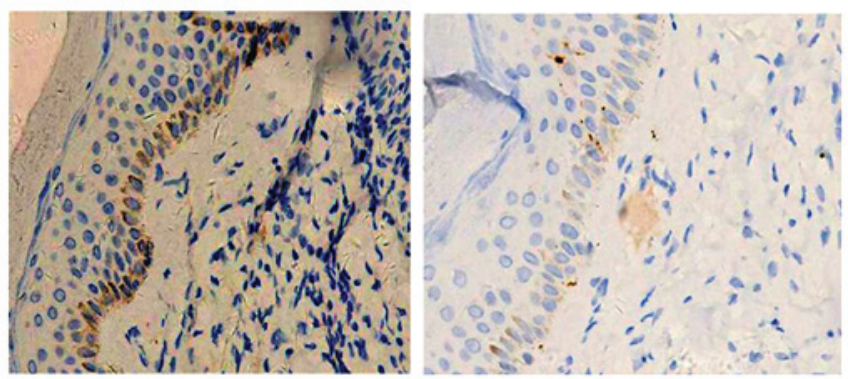

Figure 3. Immunohistochemical staining of cutaneous nodule biopsy demonstrating CD68 ( \pm ) and S-100 (-).

Literature review. In the present study, the following key words were used as search terms in PubMed: 'Multicentric reticulohistiocytosis', 'Infliximab', 'Etanercept', 'Adalimumab' and 'Tumor necrosis factor inhibition', from which 16 articles were found. According to the these reviewed articles and the results from the case described above, a total of 17 patients were treated with anti-TNF- $\alpha$ therapy, and none of the cases were excluded due to incomplete data. The present study analyzed the outcomes reported in the reviewed articles, based on the patients' responses to treatment and the reductions in steroid dosage (Table I). The data of these patients are summarized in Table I. The patients comprised 10 (62.5\%) women and six $(37.5 \%)$ men, with a median age of 47.5 years and age range of 3-76 years. All the patients developed arthritis and articular manifestations, as well as a maculopapular rash. Prior to the initiation of treatment with anti-TNF- $\alpha$ agents, the majority of the reported MRH cases had included the use of corticosteroids in their treatment, with the exception of a case reported by Iwata et al (7). Combination treatments were administered in $16(94.1 \%)$ patients in the advent of relapse and unmitigated progression of the disease. Therapeutic regimens varied in the different reports due to the absence of standardized treatment protocols. A total of $13(76.5 \%)$ patients received MTX, four (23.5\%) received cyclosporine A and eight $(47.1 \%)$ were treated with HCQ. Cyclophosphamide (CTX) was used in four cases (23.5\%) and azathioprine was used in five cases (29.4\%). A total of six patients (35.3\%) were treated with non-steroidal anti-inflammatory drugs, whereas leflunomide was used in two cases (11.8\%), and mycophenolate mofetil was used in one $(5.9 \%)$ case, as was sulfasalazine (5.9\%). A combination of chlorambucil and cariolysine was used in three cases (17.6\%). Different treatment modalities were used with little or no success prior to treatment of the patients with anti-TNF- $\alpha$ agents. Alopecia, hypoleucocytosis, pruritus and other side effects appeared following the application of immunosuppressive agents, whereas no adverse effects were reported following the use of anti-TNF- $\alpha$ agents. In the previous literature, anti-TNF- $\alpha$ agents were administered in combination with glucocorticoids in all patients with promising results, with the exception of the single case reported by Iwata et al (7). Following the initiation of anti-TNF- $\alpha$ treatment, the number of patients suffering from constitutional symptoms was relatively low. Improvements in skin lesions and arthralgia were observed upon receiving anti-TNF- $\alpha$ treatment, which indicated a positive clinical response. Only minor manifestations were found: Two (11.8\%) patients had fever, two (11.8\%) patients presented with weight loss, two (11.8\%) patients experienced fatigue, one patient $(5.9 \%)$ presented with night sweats, one patient $(5.9 \%)$ presented with stiffness and one patient $(5.9 \%)$ presented with muscle aches.

Among the cases reported in the previous studies, 10 cases included the use of etanercept for the treatment of MRH. Among these, five cases responded well to treatment (8-12), three cases reported the replacement of etanercept treatment with another anti-TNF- $\alpha$ agent, including infliximab and adalimumab. The remaining two cases reported the initiation of etanercept treatment in replacement of adalimumab (13) and infliximab (14).

In the previous literature, eight cases reported the application of infliximab for the treatment of patients with MRH, six of which reported successful treatment with infliximab $(5,14,15-18)$. The remaining two cases reported the replacement of etanercept with infliximab $(19,20)$. In one case, reported by Sellam et al (14), there was concern regarding the replacement of infliximab with etanercept.

The use of adalimumab for the treatment of patients with MRH was reported in three cases, and one case was treated successfully. Adalimumab was used in place of etanercept in one case (21), whereas two cases replaced adalimumab with etanercept (13).

\section{Discussion}

$\mathrm{MRH}$ is a rare and debilitating systemic inflammatory disease of unknown etiology. In the case of MRH, immunohistochemical analysis of synovial tissue shows positive staining of TNF- $\alpha$, IL-1 $\beta$, IL- 6 and IL-12, suggesting the presence of these inflammatory cytokines in affected areas, as reported by Gorman et al (3). In 2010, Tashiro et al (22) demonstrated the abundant accumulation of CD10 in the cytoplasm of ground-glass-like multinucleated giant cells in two patients with MRH. Of note, in a case reported by Bennàssar et al (4), increases in serum cytokine levels, namely of TNF- $\alpha$, IL-1 $\beta$, IL-6 and IL-8, were observed, which decreased following treatment.

TNF antagonists have been widely used for the treatment of rheumatoid arthritis, ankylosing spondylitis, psoriasis, psoriatic arthritis, Crohn's disease and ulcerative colitis (5,23-26). Due to the fact that high levels of TNF- $\alpha$ are expressed in patients with $\mathrm{MRH}$, anti-TNF- $\alpha$ therapy has become a viable option and widely used in the treatment of MRH in previous decades.

TNF- $\alpha$ antagonists are biological agents comprised of fusion proteins or antibodies foreign to the patient. For patients, immunogenicity and sensitization of TNF- $\alpha$ antagonists are of particular concern. The presentation of neutralized antibodies to TNF- $\alpha$ drugs can potentially cause inactivation and increased rates of clearance, thus affecting treatment outcome (27). Therefore, there were no reports pertaining to the presence of antiglobulins towards the anti-TNF- $\alpha$ agents, infliximab, etanercept and adalimumab, among the MRH cases included in the present review. In addition, there are several adverse effects of anti-TNF- $\alpha$ agents, including infusion-associated reactions, allergic reactions, increased susceptibility towards infection, demyelinating diseases and worsening of cardiovascular disease. These side effects are often mild, 


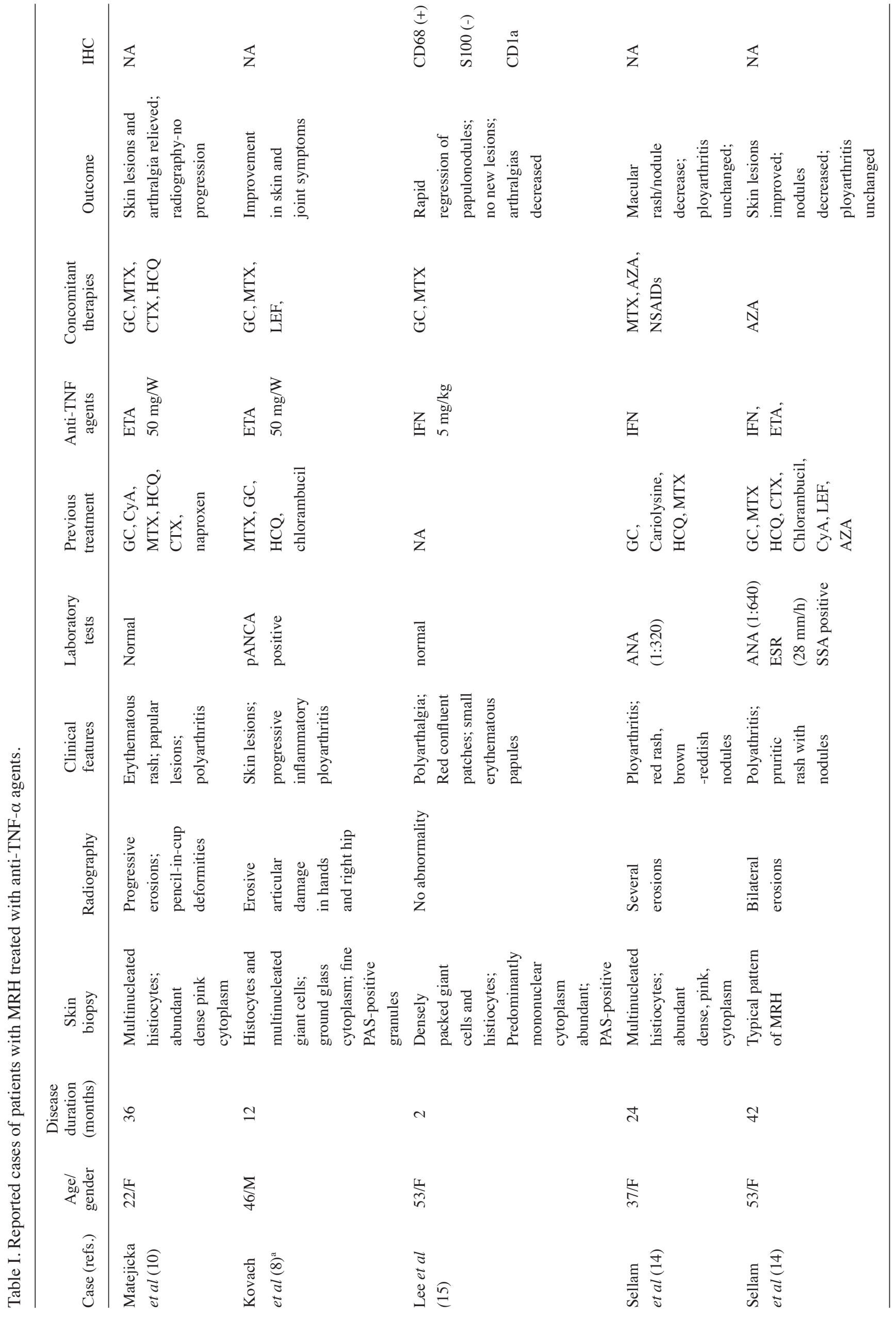




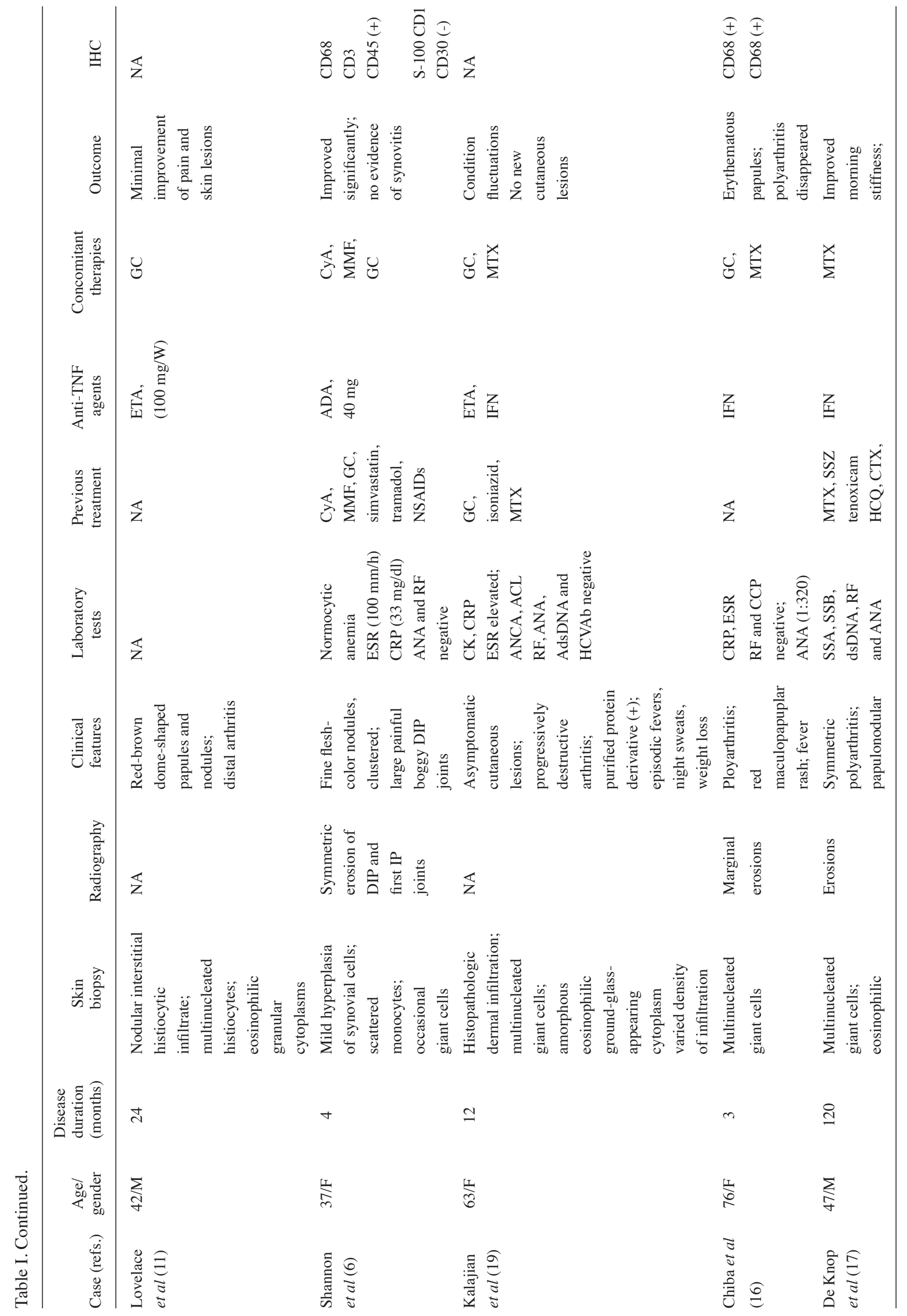




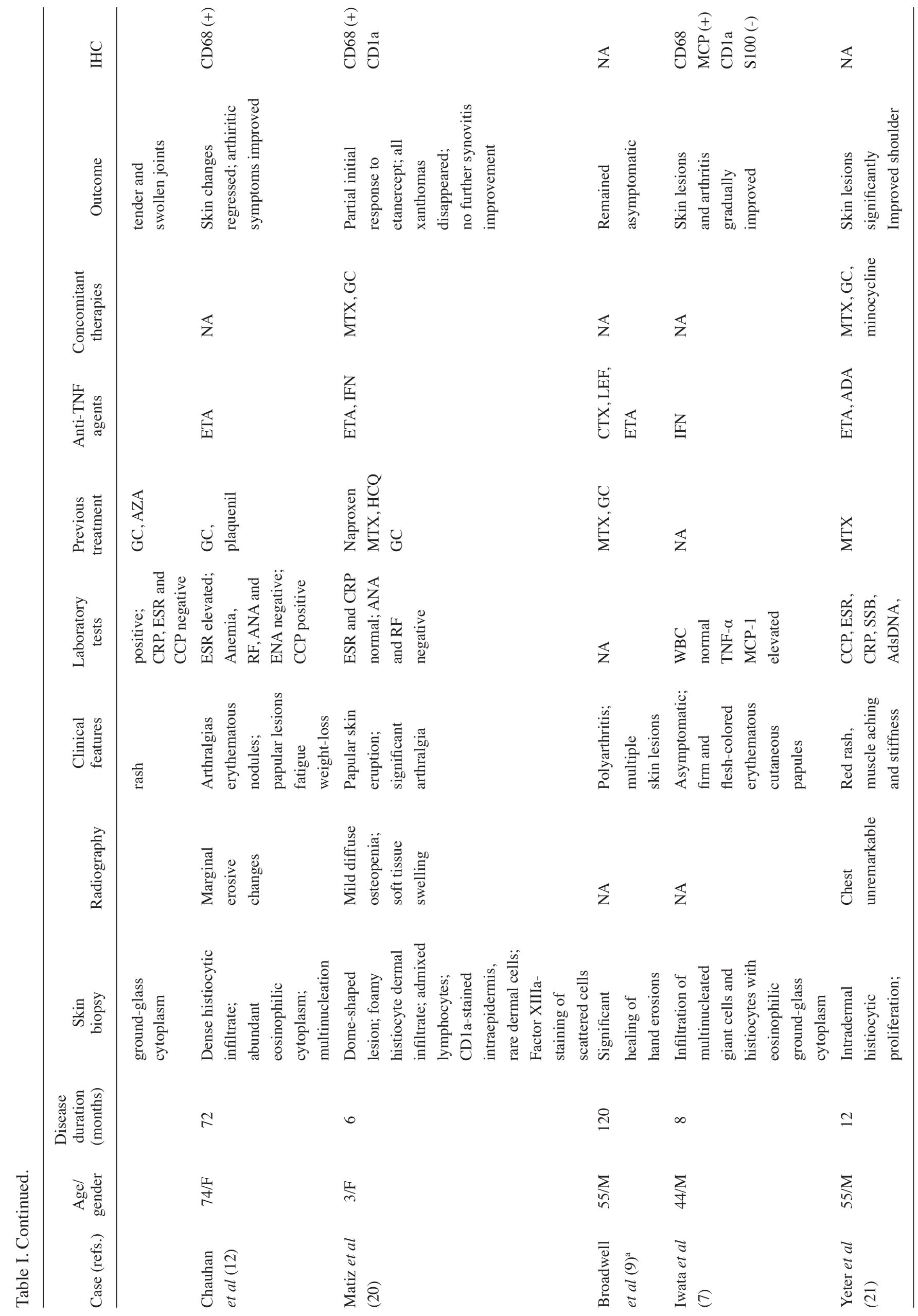




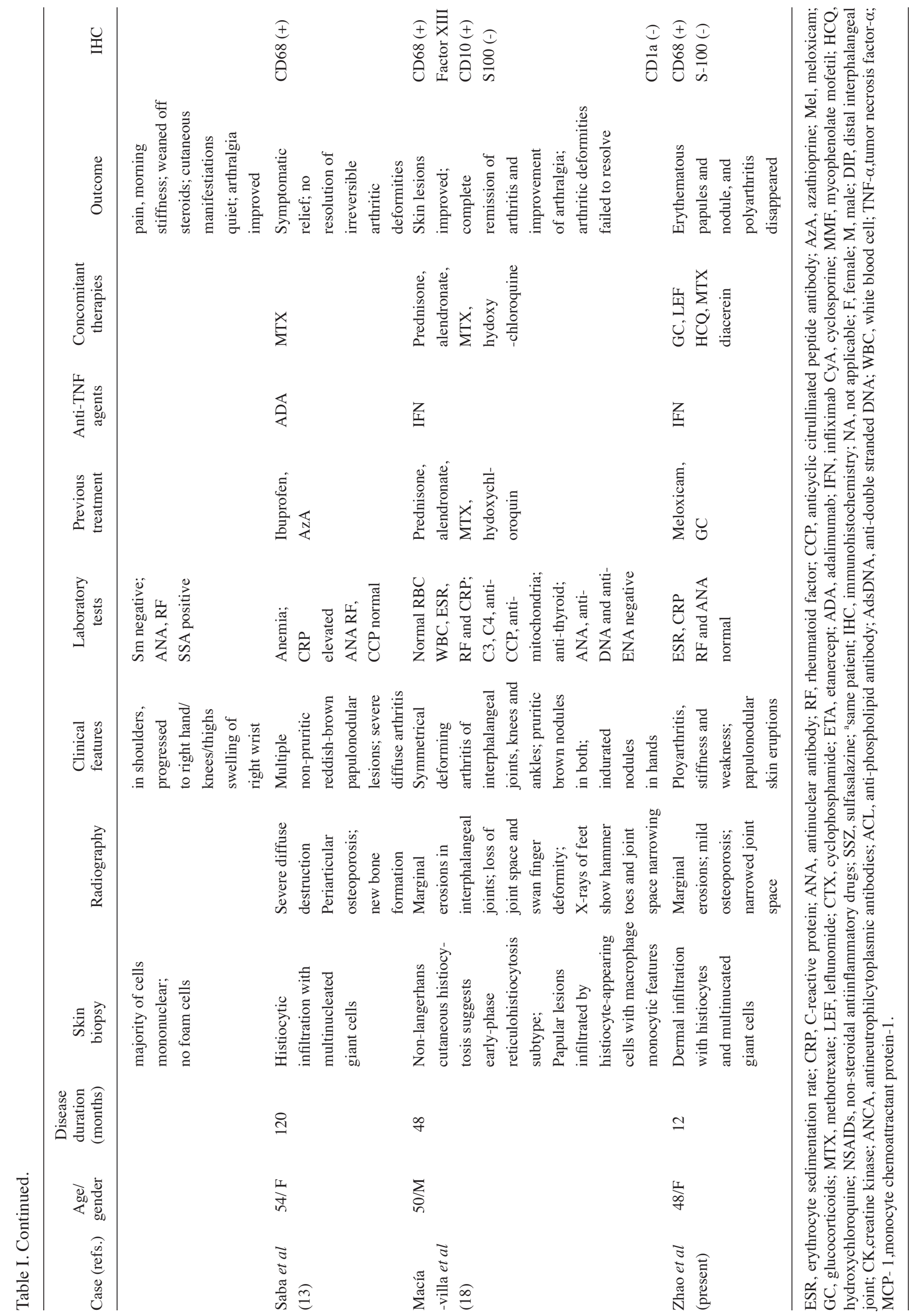


self-limiting and often do not necessitate the discontinuation of therapy (28). It is worth noting that observations or reports of these adverse effects were rare when anti-TNF- $\alpha$ treatment was used in the MRH patients in this review.

Matejicka et al performed an initial trial involving the application of anti-TNF- $\alpha$ agents in a patient with MRH in 2003. This resulted in the successful treatment of a 22-year-old female college student using a combination of etanercept (25 mg twice a week subcutaneously), MTX, prednisolone, HCQ and CTX. The patient experienced remission of skin lesions and arthralgias 6 weeks following treatment (10). The following year, Kovach et al also reported a successful case of treating MRH with the combination of etarnercept, leflunamide and prednisolone (8).

In 2004, Lee et al (15) reported an effective combination of infliximab, prednisolone and MTX in treating MRH. Treatment involved the use of infliximab ( $5 \mathrm{mg} / \mathrm{kg} / \mathrm{day})$ in combination with MTX (7.5 mg/week) and prednisolone (30 mg/day) following establishment of the diagnosis of MRH. There was a noticeable regression of dermal nodules following the first infusion, and polyarthralgia were alleviated within 3 months. Several subsequent cases of successful treatment with TNF- $\alpha$ inhibitors have been reported since, as summarized in Table I, which were effective in alleviating symptoms, although there was with disparity in responses to cutaneous and articular manifestations.

According to a review by Kalajian, a trend was noted in treatment modalities comprising TNF inhibition, with prednisolone and MTX having higher success rates $(19,29)$. Among the anti-TNF- $\alpha$ agents, infliximab has been reported to be more efficient than etanercept, which can be explained by the fact that infliximab has a higher association rate and lower dissociation rate, compared with etanercept (30). Infliximab is reported to be able to irreversibly bind to TNF without partial inhibition, thus allowing complete neutralisation of TNF (30). This may also explain the case reported by Sellam et al (14), in which no further improvement of symptoms was observed following the replacement of infliximab with etanercept. Based on the reported effectiveness of infliximab in the treatment of MRH, the patient in the present study was treated with infliximab at the beginning of treatment, which was found to be efficacious. As the patient was also receiving treatment with pednisone, MTX, leflunomide and HCQ at the same time, the combination of which has been confirmed to be effective $(3,4,29,31)$, it was not possible to independently evaluate the efficacy of infliximab in this patient (32). Thus, rather than exclusively attributing the success of treatment to infliximab, it was suggested that the inclusion of infliximab in a treatment regimen appears to be a viable option (33-35). As a result of the notable effectiveness of infliximab in treating $\mathrm{MRH}$, according to previous literature, anti-TNF- $\alpha$ agents, particularly infliximab, may be advocated as an efficacious approach for the treatment of MRH.

The present study had certain limitations, predominantly due to the rarity of the disease and the low number of patients reviewed. In addition, the administration of these TNF- $\alpha$ antagonists has often been included in various treatment regiments, however, there has been no systematic comparison between cases, and no independent evaluation of its efficiency. Furthermore, the majority of the case reports focussed partly on favorable responses, and those with poor outcomes have been rarely reported. All these limitations restrict the comprehensiveness of the analysis of anti-TNF- $\alpha$ treatment in the present study. In conclusion, as stated above, TNF antagonists offer a relatively safe and well-tolerated treatment option, and may be recommended in refractory $\mathrm{MRH}$ as they induce remission and allow a reduction in steroid dosage. It may be administered in accordance with the sequence of therapy used in the management of rheumatoid arthritis. However, further prospective investigations are required to improve and standardize its application, in terms of dosage and duration, in the treatment of patients with MRH.

\section{Acknowledgements}

This study was supported by the Hunan province Science and Technology Program (grant no. 2012SK3202).

\section{References}

1. Islam AD, Naguwa SM,Cheema GS, Hunter JC and Gershwin ME: Multicentric reticulohistiocytosis: A rare yet challenging disease. Clin Rev Allergy Immunol 45: 281-289, 2013.

2. Baek IW, Yoo SH, Yang H, Park J, Kim KJ and Cho CS: A case of multicentric reticulohistiocytosis. Mod Rheumatol 11: 1-4, 2014.

3. Gorman JD, Danning C, Schumacher HR, Klippel JH and Davis JC Jr: Multicentric reticulohistiocytosis: Case report with immunohistochemical analysis and literature review. Arthritis Rheum 43: 930-938, 2000.

4. Bennàssar A, Mas A, Guilabert A, Julià M, Mascaró-Galy JM and Herrero C: Multicentric reticulohistiocytosis with elevated cytokine serum levels. J Dermatol 38: 905-910, 2011.

5. de Vries HS, van Oijen MG, Driessen RJ, de Jong EM, Creemers MC, Kievit W and de Jong DJ: Appropriate infliximab infusion dosage and monitoring: Results of a panel meeting of rheumatologists, dermatologists and gastroenterologists. Br J Clin Pharmacol. 71: 7-19, 2011.

6. Shannon SE, Schumacher HR, Self S and Brown AN: Multicentric reticulohistiocytosis responding to tumor necrosis factor-alpha inhibition in a renal transplant patient. J Rheumatol 32: 565-567, 2005.

7. Iwata H, Okumura Y, Seishima M and Aoyama Y: Overexpression of monocyte chemoattractant protein.1 in the overlying epidermis of multicentric reticulohistiocytosis lesions: A case report. Int J Dermatol 51: 492-494, 2012.

8. Kovach BT, Calamia KT, Walsh JS and Ginsburg WW: Treatment of multicentric reticulohistiocytosis with etanercept. Arch Dermatol 140: 919-921, 2004.

9. Broadwell AW, Calamia KT, Kransdorf MJ and Ginsburg WW: Healing of erosive disease in multicentric reticulohistiocytosis. J Rheumatol 37: 1366-1367, 2010.

10. Matejicka C, Morgan GJ and Schlegelmilch JG: Multicentric reticulohistiocytosis treated successfully with an anti-tumor necrosis factor agent: Comment on the article by Gorman et al: Arthritis Rheum 48: 864-866, 2003.

11. Lovelace K, Loyd A, Adelson D, Crowson N, Taylor JR and Cornelison R: Etanercept and the treatment of multicentric reticulohistiocytosis. Arch Dermatol 141: 1167-1168, 2005.

12. Chauhan A, Mikulik Z and Hackshaw KV: Multicentric reticulohistiocytosis with positive anticyclic citrullinated antibodies. J Natl Med Assoc 99: 678-680, 2007.

13. Saba R, Kwatra SG, Upadhyay B, Mirrakhimov AE and Khan FN: Multicentric reticulohistiocytosis presenting with papulonodular skin lesions and arthritis mutilans. Case Rep Rheumatol 2013: 201563, 2013.

14. Sellam J, Deslandre CJ, Dubreuil F, Arfi S and Kahan A: Refractory multicentric reticulohistiocytosis treated by infliximab: Two cases. Clin Exp Rheumatol 23: 97-99, 2005. 
15. Lee MW, Lee EY, Jeong YI, Choi JH, Moon KC and Koh JK: Successful treatment of multicentric reticulohistiocytosis with a combination of infliximab, prednisolone and methotrexate. Acta Derm Venereol 84: 478-479, 2004.

16. Chiba E, Oda A, Tsutsumi T, Yabe H, Kamiya Y, Sakurai T, Moriguchi M, Momomura S and Terai C: Case report; a case with multicentric reticulohistiocytosis successfully treated with infliximab. Nihon Naika Gakkai Zasshi 100: 483-486, 2011.

17. De Knop KJ, Aerts NE, Ebo DG, Van Offel JF, Stevens WJ and De Clerck LS: Multicentric reticulohistiocytosis associated arthritis responding to anti-TNF and methotrexate. Acta Clin Belg 66: 66-69, 2011

18. Macía-Villa CC and Zea-Mendoza A: Multicentric reticulohistiocytosis: Case report with response to infliximab and review of treatment options. Clin Rheumatol Apr 15, 2014 (Epub ahead of print).

19. Kalajian AH and Callen JP: Multicentric reticulohistiocytosis successfully treated with infliximab: An illustrative case and evaluation of cytokine expression supporting anti-tumor necrosis factor therapy. Arch Dermatol 144: 1360-1366, 2008.

20. Matiz C, Ferguson PJ, Zaenglein A, Groh B and Bingham CA Papular xanthomas and erosive arthritis in a 3 year old girl, is this a new MRH variant? Pediatr Rheumatol Online J 7: 15 2009.

21. Yeter KC and Arkfeld DG: Treatment of multicentric reticulohistiocytosis with adalimumab, minocycline, methotrexate. Int J Rheum Dis 16: 105-106, 2013.

22. Tashiro A, Takeuchi S, Nakahara T, Oba J, Tsujita J, Fukushi J, Kiryu H, Oda Y, Xie L, Yan X, et al: Aberrant expression of CD10 in ground-glass-like multinucleated giant cells of multicentric reticulohistiocytosis. J Dermatol 37: 995-997, 2010.

23. Ma X and Xu S: TNF inhibitor therapy for rheumatoid arthritis. Biomed Rep 1: 177-184, 2013.

24. Lubrano E, Spadaro A, Amato G, Benucci M, Cavazzana I, Chimenti MS, Ciancio G, D'Alessandro G, Angelis RD, Lupoli S, et al: Tumour necrosis factor alpha inhibitor therapy and rehabilitation for the treatment of ankylosing spondylitis: A systematic review. Semin Arthritis Rheum 44: 542-550, 2015.
25. Behrens F, Cañete JD, Olivieri I, van Kuijk AW, McHugh N and Combe B: Tumour necrosis factor inhibitor monotherapy vs combination with MTX in the treatment of PsA: A systematic review of the literature. Rheumatology (Oxford) 54: 915-926, 2015.

26. Schreiber S: An update on biosimilar drugs for inflammatory bowel disease. Expert Rev Gastroenterol Hepatol 9 (Suppl 1) $: 1-3$.

27. Jung SM, Kim HS, Kim HR, Kim NY, Lee JH, Kim J, Kwok SK, Park KS, Park SH, Kim HY and Ju JH. Immunogenicity of anti-tumour necrosis factor therapy in Korean patients with rheumatoid arthritis and ankylosing spondylitis. Int Immunopharmacol 21: 20-25, 2014.

28. Scheinfeld N: A comprehensive review and evaluation of the side effects of the tumor necrosis factor alpha blockers etanercept, infliximab and adalimumab. J Dermatolog Treat 15: 280-294, 2004.

29. Trotta F and Colina M: Multicentric reticulohistiocytosis and fibroblastic rheumatism. Best Pract Res Clin Rheumatol 26: $543-557,2012$.

30. Ehlers S: Tumor necrosis factor and its blockade in granulomatous infections: Differential modes of action of infliximab and etanercept? Clin Infect Dis 41 (Suppl 3): S199-S203, 2005.

31. Lonsdale-Eccles AA, Haworth AE, McCrae FC and Young-Min SA: Successful treatment of multicentric reticulohistiocytosis with leflunomide. Br J Dermatol 161: 470-472, 2009.

32. Thalayasingam $\mathrm{N}$ and Isaacs JD: Anti-TNF therapy. Best Pract Res Clin Rheumatol 25: 549-567, 2011.

33. Pascual-Salcedo D, Plasencia C, Ramiro S, Nuño L, Bonilla G, Nagore D, Ruiz Del Agua A, Martínez A, Aarden L, Martín-Mola E and Balsa A: Influence of immunogenicity on the efficacy of long-term treatment with infliximab in rheumatoid arthritis. Rheumatology (Oxford) 50: 1445-1452, 2011.

34. Finckh A, Dudler J, Wermelinger F, Ciurea A, Kyburz D, Gabay C and Bas S; physicians of SCQM: Influence of anti-infliximab antibodies and residual infliximab concentrations on the occurrence of acquired drug resistance to infliximab in rheumatoid arthritis patients. Joint Bone Spine 77: 313-318, 2010.

35. Rosenblum $\mathrm{H}$ and Amital $\mathrm{H}$ : Anti-TNF therapy: Safety aspects of taking the risk. Autoimmun Rev 10: 563-568, 2011. 